An 8-Month-Old Girl with Prolonged Fever

Fatima Abdo, M.D., Catherine Bshouty, M.D.,

Charles W. Coffey, M.D., Robert R. Wittler, M.D.

University of Kansas School of Medicine-Wichita, Wichita, KS

\section{Department of Pediatrics}

Received Feb. 18, 2021; Accepted for publication April 1, 2021; Published online July 9, 2021 https: doi.org 10.17161 lijm.voll415208

\section{INTRODUCTION}

Francisella tularensis is an aerobic Gram-negative coccobacillus, which causes acute febrile illness of humans and other mammals, most commonly rabbits. Exposure may occur through direct contact with infected animals, arthropod bites including ticks, deer flies, and mosquitoes, contaminated soil, or ingestion of contaminated meats or water, and, in some cases, via inhalation of aerosolized organisms. Based on a retrospective study, arthropod bite was identified in $77 \%$ of cases, which was consistent with the mode of transmission in our current case. ${ }^{1}$

Manifestation of tularemia infection varies. Of the seven forms of tularemia, the classic clinical presentation is ulcero-glandular. ${ }^{1}$ Of note, each form is associated with symptoms consistent with an influenza-like illness, consisting of fevers, chills, headache, and myalgia. "Typhoidal" symptoms were reported in $47 \%$ of cases, double the proportion of the more classic manifestation of lymphadenopathy, which was contrary to the expectations.

Fever is defined as a core temperature of at least $38.0^{\circ} \mathrm{C}\left(100.4^{\circ} \mathrm{F}\right)$. Febrile illness in the pediatric population has a broad differential diagnosis. Fever can be due to infections (bacterial, viral, fungal, or tickborne), autoimmune disorders, or malignancy. A detailed history and physical examination are needed to narrow the differential diagnosis.

The following was a case of an 8-month-old presenting with fever which was found later to be due to a tick-borne illness although initial serologies for tick-borne illness were negative.

\section{CASE REPORT}

An 8-month-old girl with gross motor delay presented with febrile illness of unknown origin. The patient initially presented to a regional emergency department with lethargy, excessive sleepiness, and multiple episodes of vomiting at home for one day. At the time, parents also reported fevers of greater than $100.4^{\circ} \mathrm{F}$. The infant was admitted to the community hospital for supportive care and further evaluation. Blood and urine cultures along with a nasopharyngeal viral pathogen assay were obtained. The nasopharyngeal swab was positive for Parainfluenza 3. She was discharged following three days of hospitalization with presumptive viral illness although she remained intermittently febrile while hospitalized.

On day seven of illness, she was seen at her primary care provider's office for continued fever and was started on a course of amoxicillin to cover empirically for possible bacterial infection. However, persistent fevers, decreased oral intake, and decreased number of wet diapers prompted parents to have her re-evaluated at the local emergency department on day 11 of illness. In the emergency room, the patient was noted to have a scar with a scab at the left postauricular area where a tick had been removed. The scar had not been visualized at the initial emergency department visit (Figure 1).

\section{KANSAS JOURNAL of MEDICINE}

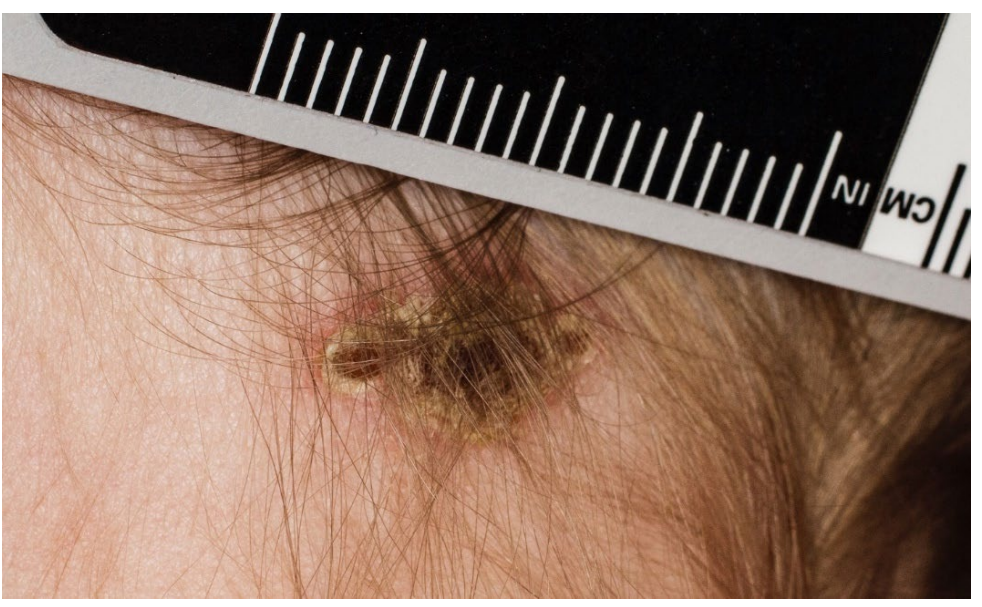

Figure 1. Left postauricular tick bite site.

Of note, there was no recent travel outside of the United States, however, the family was in Colorado a month prior to her illness. The patient was exposed to a cat and a dog at home, but there was no recollection of an animal bite or scratch. The infant's past medical history was noncontributory, and immunizations were up to date.

Given the clinical findings, the patient was admitted for parenteral antimicrobial therapy for suspected tick-borne disease with doxycycline. Pertinent lab findings from the regional hospital included an elevated white blood count of $19.1 \mathrm{~K} / \mathrm{cumm}$ (5.0 - 18.0), decreased Hgb level of $8.6 \mathrm{gm} / \mathrm{dL}$ (11.0 - 14.0), elevated platelet count of $630 \mathrm{~K} / \mathrm{cumm}$ (150 - 450), elevated liver enzymes of AST $463 \mathrm{IU} / \mathrm{L}(0$ - 40) and ALT of $202 \mathrm{IU} / \mathrm{L}(0-30)$, as well as a decreased sodium level of $126 \mathrm{mmol} / \mathrm{L}$ (135 - 148), and decreased albumin of $2.1 \mathrm{~g} / \mathrm{dL}$ (4.1 - 5.2). Serologic assays were obtained for Epstein Barr Virus, Cytomegalovirus, Bartonella henselae, Francisella tularensis, and Rickettsia ricketsiia. The patient was transferred to a tertiary care center for further management on day four of hospitalization due to persistent symptoms on day 14 of illness.

On admission to the tertiary care center, the patient remained on IV doxycycline. Significant lab findings on admission were remarkable for elevated lactate dehydrogenase of 931 units/L (reference range 180 430), low uric acid of $1.1 \mathrm{mg} / \mathrm{dL}$ (2.6 - 6.0), elevated c-reactive protein of $123 \mathrm{mg} / \mathrm{L}(<8.0)$, increased prothrombin time of $16.6 \mathrm{sec}(9.8-13.0)$, and a low hemoglobin of $7.4 \mathrm{gm} / \mathrm{dL}$ (11.0 - 14.0). Pertinent imaging included a CT of the abdomen which showed multiple hypo-attenuated foci in the spleen and a CT of the pelvis which showed prominent inguinal lymph nodes. Flow cytometry on peripheral blood obtained at the time did not show any evidence of leukemia. The above noted serologic assays from the referring hospital were all negative.

The patient continued to be febrile despite six days of therapy with IV doxycycline. Due to suspicion for tularemia infection, antimicrobial therapy was switched to IV gentamicin. The initial serology for tularemia, which was obtained at day 14 of illness, was negative. The patient was treated for an additional six days on IV gentamicin. After being afebrile for greater than 24 hours, the patient was discharged home 
KANSAS JOURNAL of MEDICINE PROLONGED FEVER continued.

with a 10-day course of ciprofloxacin. Prior to discharge on day 21 of illness, tularemia antibody was re-tested and was positive at a titer of 2560 (negative $<20$ ).

\section{DISCUSSION}

Our case presented with the less common manifestation of the typhoidal form of tularemia based on her clinical symptoms of persistent fever, no apparent lymphadenopathy or lymphadenitis, no pharyngitis, and no pneumonia. ${ }^{1}$ Ulcero-glandular tularemia is the most frequent clinical presentation of tularemia which presents with an ulcerative lesion at the inoculation site (tick or animal bite) and a regional lymphadenitis. Tularemia is particularly endemic to areas in south-central United States and most frequently is transmitted by ticks rather than by animals such as rabbits. There are many limiting factors in diagnosing tularemia; its clinical presentation is nonspecific and similar to other more frequent summer febrile illnesses such as enterovirus infection. Other differential diagnoses for prolonged fever include rheumatologic diseases or malignancy.

Antibodies to Francisella tularensis often remain negative for the first 7 to 14 days of infection as observed in our patient. ${ }^{2}$ Those factors contribute to a prolonged time to diagnosis and a delay in appropriate treatment for tularemia. For that reason, in the pediatric population, it is imperative to have a high suspicion of typhoidal tularemia infection in the setting of a febrile illness even without lymphadenopathy, especially during tick season in an endemic region., ${ }^{1,2}$ Complications associated with tularemia include pneumonia, sepsis, and meningitis. ${ }^{1}$

Interestingly, the CT scan of our patient's abdomen was significant for hematogenous spread with focal lesions involving the spleen. A PubMed (pubmed.gov) search of tularemia, human, and splenic lesion or splenic nodule found only one prior case. ${ }^{3}$ Imaging of the liver and spleen should be considered in cases of fever of unknown origin given the possibility of tularemia and hepatosplenic Bartonella henselae infection.

Early detection or suspicion of tularemia and initiation of appropriate antibiotic therapy plays a significant role in success of treatment. Antibiotics such as ceftriaxone or other beta-lactam antibiotics that commonly are given for empiric treatment of children with persistent fever have not been shown to be effective treatment for tularemia. Aminoglycosides, such as streptomycin or gentamicin, are first line drugs of choice. ${ }^{4}$ Doxycycline is the drug of choice for tickborne Rickettsial infections and monocytic erlichiosis which have some overlapping presentations with typhoidal tularemia, however, concern for relapse occurs when used as monotherapy for tularemia. ${ }^{5}$ Although aminoglycosides are effective in treating tularemia, they only can be administered parentally, requiring prolonged hospitalization. In addition, the toxicity associated with the use of aminoglycosides is of concern. ${ }^{6}$ In comparison to gentamicin, streptomycin has been shown to be more effective in the treatment of oropharyngeal tularemia. ${ }^{7}$ Patients with mild to moderate cases of tularemia have been treated successfully with oral ciprofloxacin. ${ }^{8}$ Aminoglycosides should be given for 7 - 10 days, whereas ciprofloxacin would be given for 10 - 14 days. $^{6}$

Our patient was switched from intravenous doxycycline to intravenous gentamicin on day 15 of illness due to a high suspicion for tularemia despite negative initial serological tests for tularemia, which was obtained on day 11 of illness. Our patient had clinical improvement within 24 hours of initiating gentamicin and remained on gentamicin therapy for a total of six days prior to transitioning to oral therapy with ciprofloxacin. As mentioned earlier, repeated serology for tularemia at day 19 of illness was positive with a titer of 2560 confirming the diagnosis of tularemia.

Tick-borne illness should be considered as part of a broad differential diagnosis for fever of unknown origin, especially in endemic regions within the pediatric population. Similar to Rocky Mountain Spotted Fever, tularemia antibodies may not be detected within the first 14 days of illness and sometimes may take up to 21 days to be detected. ${ }^{2}$ Since early antibiotic treatment decreases the morbidity associated with tularemia, a high index of suspicion should prompt early antibiotic use even in the absence of positive serology. It is worth noting that the clinical presentation of tularemia may be variable and may present with unusual manifestations, such as the splenic involvement as seen in our case.

\section{REFERENCES}

${ }^{1}$ Lester Rothfeldt LK, Jacobs RF, Wheeler JG, Weinstein S, Haselow DT. Variation in tularemia clinical manifestations-Arkansas, 2009-2013. Open Forum Infect Dis 2017; 4(1):ofx027. PMID: 28480295.

${ }^{2}$ Snowden J, Stovall S. Tularemia: Retrospective review of 10 years' experience in Arkansas. Clin Pediatr 2010; 50(1):64-68. PMID: 20837613.

${ }^{3}$ Garver MK, St. Geme JW, Siegel MJ. Tularemia presenting with splenic nodules. Pediatr Infect Dis J 1994; 13(9):830-832. PMID: 7808857.

4 Nigrovic LE, Wingerter SL. Tularemia. Infect Dis Clin North Am 2008; 22(3):489-504. PMID: 18755386.

${ }^{5}$ Caspar Y, Maurin M. Francisella tularensis susceptibility to antibiotics: A comprehensive review of the data obtained in vitro and in animal models. Front Cell Infect Microbiol 2017; 7:122. PMID: 28443249.

${ }^{6}$ Kimberlin DW, Brady MT, Jackson MA, Long SS, American Academy of Pediatrics. Committee on Infectious Diseases. Red Book: 2018-2021 Report of the Committee on Infectious Diseases. American Academy of Pediatrics; 2018.

7 Kaya A, Uysal IO, Guven AS, et al. Treatment failure of gentamicin in pediatric patients with oropharyngeal tularemia. Med Sci Monit 2011; 17(7):CR376-CR380. PMID: 21709631.

8 Johansson A, Berglund L, Gothefors L, Sjostedt A, Tarnvik A. Ciprofloxacin for treatment of tularemia in children. Pediatr Infect Dis J 2000; 19(5):449-453. PMID: 10819342.

Keywords: tick-borne disease, Francisella tularensis, tularemia, fever of unknown origin, splenic disease 